Commun. math. Phys. 20, 1-8 (1971)

(C) by Springer-Verlag 1971

\title{
Collisionless Radiation in Closed Cosmologies
}

\author{
RichARD A. MATZNER \\ Institute of Theoretical Astronomy, University of Cambridge, Cambridge, England \\ and Department of Physics, University of Texas, Austin Texas 78712, USA
}

Received August 14, 1970

\begin{abstract}
An outline is given of some new results for collisionless radiation in Bianchi type IX cosmologies. An interesting new effect is displayed which distorts the pattern of any microwave temperature anisotropy from the quadrupole + dipole shape previously predicted in these models.
\end{abstract}

\section{Introduction}

This paper presents an outline of several new results for rotating homogenous anisotropic cosmologies containing collisionless radiation. A fuller account has been submitted for publication elsewhere.

We concentrate on Bianchi type IX homogeneous cosmologies [1-5] which contain collisionless radiation ("neutrinos") and in which there may be rotation. A type IX metric may be written [3]

$$
d s^{2}=-d t^{2}+R^{2}(t) e_{i j}^{2 \beta(t)} \sigma^{i} \sigma^{j},
$$

where $\beta_{i j}$ is a $3 \times 3$ symmetric traceless matrix, not in general diagonal, and the $\sigma^{i}$ obey the curl relations of type IX $[2,3]$ :

$$
d \sigma^{i}=\frac{1}{2} \varepsilon_{i j k} \sigma^{j} \wedge \sigma^{k} .
$$

In dealing with collisionless radiation we write the homogenous stress tensor [6-8]

$$
T_{\mu \nu}=R^{-3} \int F\left(q_{i}, t\right) q_{\mu} q_{v}\left(q^{0}\right)^{-1} d^{3} q
$$

where the components $\mathrm{q}_{\alpha}$ are expressed in the $\left\{d t, \sigma^{i}\right\}$ basis of Eq. (1.1) and $\left(q^{0}\right)^{2}=R^{-2} e_{i j}^{-2 \beta} q_{i} q_{j}$. Also, $d^{3} q=d q_{1} d q_{2} d q_{3}$.

For a collisionless system $F\left(q_{i}, t\right)$ is a solution of the collisionless Boltzmann equation, and hence is a function only of constants of the geodesic motion [7]. $F$ therefore can be expressed solely as a function of the three quantities $k_{i}$ :

$$
k_{i} \equiv q_{i}\left(t_{0}\right)
$$

1 Commun. math Phys., Vol. 20 
which are simply the values of the momentum components at some "initial time" $t_{0}$. The geodesic equation is [9]

$$
\frac{d q_{i}}{d t}+\frac{\varepsilon_{i j k} q_{j} q^{k}}{q^{0}}=0
$$

where $q^{k}=R^{-2} e_{k l}^{-2 \beta} q_{l}$. According to Eq. (1.5) $q^{2} \equiv q_{i} q_{i}$ is constant, so one has

$$
q^{2}=k^{2} \equiv k_{i} k_{i} .
$$

A less obvious result from Eq. (1.5) is that the Jacobian

$$
J\left(q_{i}, t ; k_{j}\right)=1 ;
$$

that is, the volume integration element is conserved:

$$
d^{3} q=d^{3} k .
$$

Combined with Eq. (1.6), this shows that the angular integration element is also conserved:

$$
d n_{3} d \phi=d m_{3} d \phi_{k}
$$

where $n_{i}=q_{i} / q, \phi=\arctan \left(q_{1} / q_{2}\right), m_{i}=k_{i} / k$ and $\phi_{k}=\arctan \left(k_{1} / k_{2}\right)$.

Because the only nontrivial coordinates for the neutrino motion are $n_{3}$ and $\phi$, it is important to realize that the conservation of the simplectic form $d n_{3} \wedge d \phi$ by the motion implies the existence of a Hamiltonian. In the Hamiltonian formulation $n_{3}$ and $\phi$ are the coordinate and conjugate momentum in a time dependent 1 -dimensional Hamiltonian system. The Hamiltonian is found to be

$$
H=n^{0} \equiv R^{-1}\left(e_{i j}^{-2 \beta} n_{i} n_{j}\right)^{\frac{1}{2}} .
$$

The "phase space" for this Hamiltonian system is the unit sphere of neutrino directions.

The form of the integral in Eq. (1.3) for $T_{00}$ and the corresponding form for the spatial stresses shows that

$$
\stackrel{\circ}{T}_{i j}-\frac{1}{3} \delta_{i j}{\stackrel{\circ}{T_{k}}}=-\frac{\partial T_{00}}{\partial \beta_{i j}}
$$

where one holds all quantities except $\boldsymbol{\beta}$ constant in the derivative on the right. The quantities $\stackrel{\circ}{T}_{i j}$ are the components of the stress tensor expressed in an orthonormal tetrad $\left\{d t, \omega^{i}\right\}$ with $\omega^{i} \equiv R^{-1} e_{i j}^{-\beta} \sigma^{j}$. Eq. (1.10) is analogous to one found by Misner [6] for type I.

None of the results in this section have made use of the field equations. 


\section{The Lagrangian Formulation}

We consider a distribution function describing neutrinos which were thermal at one instant (the production, or decoupling, instant). At that instant they had a fluid-like stress tensor in their rest frame, and this rest frame had a definite velocity $U^{\alpha}$. The distribution function was thus of the form $F\left(p_{\alpha} U^{\alpha} / T_{d}\right)$, where $T_{d}$ is the temperature at the decoupling instant, and $p_{\alpha}$ (the components of the momentum at the decoupling instant) and $U_{\alpha}$ are henceforth to be expressed in the $\left\{d t, \omega^{2}\right\}$ frame. That is,

$$
p^{i}=R_{d}^{-1} e_{i j}^{-\beta^{d}} k_{j} ; \quad\left(p^{0}\right)^{2}=R_{d}^{-2} e_{i j}^{-2 \beta^{d}} k_{i} k_{j},
$$

where we take the decoupling instant $t_{d}$ as the initial time and $k_{i}=q_{i}\left(t_{d}\right)$ as the initial conditions for the subsequent evolution of the problem. Also $R_{d} \equiv R\left(t_{d}\right)$ and $\boldsymbol{\beta}^{d} \equiv \boldsymbol{\beta}\left(t_{d}\right)$ Rotation can be shown to be equivalent to non zero $U_{i}$.

Substituting Eqs. (2.1) into Eq. (1.3) for the stress tensor, and partially evaluating the resultant integral one obtains

Here

$$
T_{00}=\varrho_{v}\left[1+V_{v}(\boldsymbol{\beta}, t)\right] .
$$

$$
\varrho_{v}=a_{v}\left(T_{d} R_{d} / R\right)^{4}
$$

where $a_{v}$ is the "Stefan-Boltzmann" constant appropriate to the type of particles under consideration, and $T_{d} R_{d} / R$ is the adiabatic law for the decrease of temperature. The quantity $V_{v}$ is defined by

$4 \pi\left[1+V_{v}(\boldsymbol{\beta}, t)\right]=\int\left(e_{i j}^{-2 \beta} n_{i} n_{j}\right)^{\frac{1}{2}}\left[U^{0}\left(e_{a b}^{-2 \beta^{d}} m_{a} m_{b}\right)^{\frac{1}{2}}-U_{c} e_{c d}^{-\beta^{d}} m_{d}\right]^{-4} d n_{3} d \phi$.

The $U^{0}$ and $U_{i}$ are the components in the $\left\{d t, \omega^{i}\right\}$ frame.

Eq. (2.4) for the "potential" $V_{v}$ is analogous to one defined by Misner [6] for type I, where the denominator in Eq. (2.4) reduces to unity. Because the denominator of Eq. (2.4) is bounded above and below one has immediately that

$$
D_{-}^{-1} V_{v I}(\boldsymbol{\beta}) \geqq V_{v}(\boldsymbol{\beta}, t) \geqq D_{+}{ }^{-1} V_{v I}(\boldsymbol{\beta}),
$$

where $V_{v I}$ is Misner's type I potential [6] and $D_{ \pm}$are the bounds on the denominator in Eq. (2.4). Now for large $|\beta|, V_{v I}$ is exponentially steep, so the potential $V_{v}(\boldsymbol{\beta}, t)$ has equipotentials which for large anisotropy are similar to those of $V_{v I}(\boldsymbol{\beta})$.

By considering the implications of the conservation of the number of neutrinos, one can prove the following inequality:

$$
V_{v}(\boldsymbol{\beta}, t) \geqq\left(U^{0}\right)^{4 / 3}-1 .
$$

Since the proof is rather long, we shall not give it here. 
In view of Eq. (1.10), and techniques developed by Misner $[3,6]$ one concludes that the

$$
\stackrel{\circ}{T}_{i j}-\frac{1}{3} \delta_{i j} \stackrel{\circ}{T}_{k k}=\stackrel{\circ}{G}_{i j}-\frac{1}{3} \delta_{i j} \stackrel{\circ}{G}_{k k}
$$

equations can be obtained by the variation of $\beta$ in the Lagrangian

where

$$
\mathscr{L}=R^{3}\left[\frac{1}{2} \sigma_{i j} \sigma_{i j}-\varrho_{g} V_{g}(\boldsymbol{\beta})-\varrho_{v} V_{v}(\boldsymbol{\beta}, t)\right],
$$

$\sigma_{i j}=\frac{1}{2}\left[\left(e^{\beta}\right)^{\cdot} e^{-\beta}+e^{-\beta}\left(e^{\beta}\right)\right]_{i j} \quad$ (the dot means time derivative); and

$\varrho_{g}=\frac{3}{4} R^{-2},(R$ is taken as a given function of time $)$,

$$
V_{g}(\boldsymbol{\beta})=\frac{1}{3} \operatorname{trace}\left(e^{4 \beta}-e^{-2 \beta}+\mathbf{1}\right) .
$$

It is possible to parametrize the matrix $\boldsymbol{\beta}$ by giving the Euler angles $\varphi, \theta, \psi$ defining the orientation of the principal axes of the metric, and two parameters, $\beta_{+}$and $\beta_{-}$which are linear combination of the eigenvalues of $\boldsymbol{\beta}$. (Recall $\boldsymbol{\beta}$ is traceless.) Because the trace operation is rotation invariant, $V_{g}$ is independent of $\varphi, \theta$, and $\psi$.

Misner [6] has shown that in the large anisotropy non-rotating case, the system point, described by the parameters $\beta_{ \pm}$, obeys

$$
\left|\frac{d \beta_{+}}{d \Omega}\right|^{2}+\left|\frac{d \beta_{-}}{d \Omega}\right|^{2} \leqq 1,
$$

where $\Omega=-\ln \left(R / R_{\max }\right)+$ const. In the rotating case Eq. (2.9) still holds. Additionally Misner [6] has shown that $V_{v I}$ has equipotential contours of triangular shape, and that the position of a "wall" due to $V_{v I}$ (i.e. a region where $V_{v I}$ becomes effective in affecting the motion) moves outward with a $(d / d \Omega)$ speed of order unity as $\Omega$ increases (i.e. as the model nears the singularity). Because of Eq. (2.5) a similar result holds in type IX for $V_{v}(\boldsymbol{\beta}, t)$.

Let us now consider the evolution of the model as $\Omega$ increases, approaching the singularity. We assume that the anisotropy is large. (Roughly this means that the position of the potential walls, $\beta_{w}$, satisfies $\beta_{w} \widetilde{\sim}$ ). From the analysis above we see that the system point can reach the $V_{v}(\beta, t)$ wall at most once during the large anisotropy regime. Misner has shown that such collisions result in a quick reflection after which the system point moves in the opposite direction from the wall. (Misner [6] dealt with type I but because of Eq. (2.5) the result applies here.) Because of the triangular contours of $V_{v}$, the system point can never again reach a wall of $V_{v}(\beta, t)$. Hence the entire large anisotropy evolution after the single encounter is dominated by the potential $V_{g}$. But $V_{g}$ is independent of $\theta, \varphi$, and $\psi$ so $\mathscr{P}$, the total angular momentum formed in the usual 
way from the momenta conjugate to the Euler angle coordinates, is constant throughout most of the large anisotropy evolution.

Because the $V_{v}$ potential does not affect the motion after the single encounter, the results of Misner [10] for mixing in non-rotating models apply to these models as well. Rotation does not interfere with mixing.

\section{Small Anisotropy Calculations; the Microwave Temperature Anisotropy}

If one assumes that some sort of dissipative process such as the neutrino viscosity suggested by Misner [6] reduces the anisotropy $\boldsymbol{\beta}$ (and its derivatives) and the quantities $\left|U_{i}\right|$ to small values by the decoupling epoch (in a discussion of $e$-neutrinos this is a temperature of $\sim 10^{10} \mathrm{~K}$ ), then a small anisotropy calculation can be made.

The potential $V_{g}$ in that case becomes negligible in the Lagrangian because $\varrho_{g}$ is small compared to $\varrho_{v}$. (Only at the present epoch, when $T \sim 3 K$, is $\varrho_{g} \propto R^{-2}$ perhaps comparable to $\varrho_{v} \propto R^{-4}$.) In the small anisotropy limit, $V_{v}$ reduces to the $V_{v I}$ defined by Misner (to within a constant additive factor):

$$
V_{v}=\frac{4}{15} \hat{\beta}_{i j} \hat{\beta}_{i j}+\text { const. }
$$

where $\hat{\boldsymbol{\beta}}=\boldsymbol{\beta}-\boldsymbol{\beta}^{d}$. This simple-harmonic form can be exploited in the Lagrangian, which reduces to

$$
\frac{1}{3} \mathscr{L}=R^{3}\left\{\dot{\beta}_{Y} \dot{\beta}_{Y}-\frac{8 \varrho_{v}}{15}\left[\left(\beta_{1}-\beta_{1}^{d}\right)^{2}+\left(\beta_{2}-\beta_{2}^{d}\right)^{2}+\sum_{Y=3}^{5} \beta_{Y}^{2}\right]\right\},
$$

where $\boldsymbol{\beta}$ is parametrized:

$$
\boldsymbol{\beta}=\left[\begin{array}{ccc}
\beta_{1}+\sqrt{3} \beta_{2} & \sqrt{3} \beta_{3} & \sqrt{3} \beta_{4} \\
\cdot & \beta_{1}-\sqrt{3} \beta_{2} & \sqrt{3} \beta_{5} \\
\cdot & \cdot & -2 \beta_{1}
\end{array}\right] .
$$

The decoupling value, $\boldsymbol{\beta}^{d}$, can be considered diagonal so only $\beta_{1}^{d}$ and $\beta_{2}^{d}$ are nonvanishing.

A Lagrangian of the form (3.2) is analogous to the small anisotropy one found in type I by Misner [6], except that here we have more variables, describing the possibility that the principal axes may rotate. We find solutions similar to Misner's type I solutions.

While the model is radiation dominated, if the neutrinos are not too small a fraction of the matter present, the $\beta_{Y}, Y=1 \ldots 5$ oscillate with amplitude $\propto R^{-\frac{1}{2}}$ about the point $\beta_{Y}^{d}$.

If however, one supposes that there is also some baryon matter present, then the radiation density $\varrho \propto R^{-4}$ will eventually drop below 
the baryon matter density, $\varrho_{b} \propto R^{-3}$. In realistic models this will occur at temperatures of $\sim 3 \times 10^{4} \mathrm{~K}$ (high density model) to $3 \times 10^{2} \mathrm{~K}$ (low density model).

After the model becomes baryon-matter dominated, again in analogy with Misner's [6] results, we find that the $\beta_{Y}$ motion is strongly damped (because of the different time dependence of $R$ ). We find

$$
\frac{d \beta_{Y}}{d \Omega} \propto\left(R / R_{1}\right)^{-3 / 2}
$$

where the subscript " 1 " indicates the value of a quantity at the instant $t_{1}$ when the matter first became dominant. We conclude that subsequent to $t_{1},\left(\beta-\beta^{d}\right)_{Y}$ is essentially constant at a value of the order of its value at $t_{1}$.

It is not difficult to show that the observed directional temperature of these neutrinos, or of photons, which behave similarly once the universe becomes transparant, has the form:

$$
\frac{T\left(s_{i}\right)}{T_{0}}=1+\left(\beta^{d}-\beta\right)_{c d} s_{c} s_{d}+U_{i} s_{i}+\cdots .
$$

Here $s_{i}$ are the direction cosines of the momentum of photons being observed and $T_{0}$ is a mean temperature. The quadrupole term $\left(\beta-\beta^{d}\right)_{i j} s_{i} s_{j}$ is of order $\left|\beta-\beta^{d}\right|$ and the dipole (Doppler) term $U_{i} s_{i}$ is of the order of $\left|U_{i}\right|$ if the observer is at rest in the homogeneous frame. The observed smallness of the blackbody anisotropy [11] implies that both these terms are of the order of $10^{-3}$ or smaller. It has therefore been assumed that the next order terms will be order $(\Delta T / T)^{2}$ and hence unobservable. However, a careful analysis of the temperature anisotropy shows that there are some second order terms of the form $|\beta|\left|\beta-\beta^{d}\right|$. The quantity $\beta$ is invariantly defined because it appears in $V_{g}$ and need not be small. The only observational determination of $|\beta|$ so far is Sandage's [12] result that the decelleration parameter suggests a closed universe. One writes the expansion law (see $[4,5$, or 6$]$ ):

$$
3(\dot{R} / R)^{2}=T_{00}+\frac{1}{2} \sigma_{i j} \sigma_{i j}+\varrho_{g}\left(V_{g}-1\right) .
$$

The Robertson-Walker form has $V_{g}=0$. If $V_{g}>1$ then the $k=+1$ term (i.e. $-\varrho_{g}=-\frac{3}{4} R^{-2}$ ) in Eq. (3.6) is swamped, and the "closed" behaviour suggested by Sandage's observations cannot appear.

The contour $V_{g}=1$ stretches off to infinity in some directions, but for $V_{g}<1$ the enclosed region is bounded (in the $\beta_{+}-\beta_{-}$plane). For definiteness one may take $|\beta| \sim 0.1$ which does not violate our small $|\beta|$ approximation too severely, but which leads to sizeable effects, as we show below. From our analysis above we see that $\boldsymbol{\beta}$ has been essentially constant since the instant $t_{1}$ that the model became matter dominated. 
Suppose we define an evolution matrix $A_{i j}\left(m_{b}, t\right)$ :

$$
m_{i}=A_{i j}\left(m_{b}, t\right) n_{j}
$$

which connects the "direction cosines" $n_{i}$ of a particular photon (or neutrino) with their initial values $m_{i}$. Obviously $A_{i j}$ is an orthogonal matrix. The freedom of rotations about $m_{i}$ or $n_{i}$ is not important. $A_{i j}$ is a function both of the particular photon considered and of the time. One finds:

$$
T_{0} / T\left(s_{i}\right)=1-\left(A^{T} \beta^{d} A-\beta\right)_{c d} s_{c} s_{d}-U_{i} A_{i j} s_{j}+\text { terms of order }(\Delta T / T)^{2} .
$$

The term $U_{i} A_{i j} s_{j}$ is a Doppler shift term corrected for the direction of the incident flux. The term $\left(A^{T} \beta^{d} A-\beta\right)_{c d} S_{c} S_{d}$ gives the corrected quadrupole pattern, and measures the difference between the decoupling anisotropy $\boldsymbol{\beta}^{d}$, as presented by the transformed direction of the photons, and the current value $\boldsymbol{\beta}$.

It is not necessary to use $A_{i j}$ explicitly in what follows; one simply finds the solutions to the geodesic equation to lowest order in $\boldsymbol{\beta}$. These are

$$
\begin{gathered}
\phi=\phi_{k}-m_{3} \int d \eta\left[6 \beta_{+}-2 \sqrt{3} \beta_{-} \cos \left(2 \varphi+2 \phi_{k}\right)\right], \\
n_{3}=m_{3}-\left(1-m_{3}^{2}\right) \int d \eta 2 \sqrt{3} \beta_{-} \sin \left(2 \varphi+2 \phi_{k}\right),
\end{gathered}
$$

in a model which has only a 1-2 off-diagonal component [5]:

In Eqs. (3.9)

$$
\beta_{1}=\beta_{+}, \quad \beta_{2}=\beta_{-} \cos 2 \varphi, \quad \beta_{3}=\beta_{-} \sin 2 \varphi .
$$

$$
d \eta=R^{-1} d t
$$

In typical models, the interval $\Delta \eta$ between the last photon scattering and the "current" epoch $(T \sim 3 K)$ is $\Delta \eta \sim 0.5$ to 1.5. Also, since the baryon matter has been dominant for most (or all) of the time since the last photon scattering, $\beta_{+}, \beta_{-}$and $\varphi$ have been essentially constant as the photons traveled.

Eqs. (3.9) show that the resultant directions of the photons will not lead to a quadrupole temperature pattern, but instead the pattern will resemble one which has been torqued or twisted about each of the principal axes. Hence the locus of directions of maximum temperature in the sky will no longer be a great circle but will be some more complicated (roughly $S$-shaped) curve. If one estimates $|\beta| \sim 0.1, \Delta \eta \sim 0.5$ we see the angles of twisting are small $\Delta \phi \sim 0.1$. If however, one allows $|\beta| \sim 1$ then $\Delta \phi \sim 1$ and the quadrupole shape could be completely destroyed. (Of course $|\beta| \sim 1$ violates our small anisotropy assumption but the result is suggestive.)

These twisting effects appear even in non-rotating models. In type IX models, rotation is intimately connected to the size of $U_{i}$ [9]. The ob- 
servational limits on the dipole anisotropy exclude sizeable rotation so effects specifically due to rotation in realistic models are too small to be important.

This work was supported in part by NSF grants GU 2630 (to Wesleyan University where this work was begun) and GP 20033 (University of Texas).

\section{References}

1. Bianchi, L.: Lezioni Sulla Teoria dei Gruppi Continui Finiti, Pisa: Spoerri 1918.

2. Taub, A. H.: Ann. Math. 53, 474 (1951).

3. Misner, C. W.: Essay submitted to gravity foundation competition (unpublished, 1967).

4. Matzner, R. A., Shepley, L. C., Warren, J. B.: Ann. Phys. (N.Y.) 57, 401 (1970).

5. Ryan, M. P.: Ph.D. thesis, University of Maryland, Department of Physics and Astronomy (unpublished, 1970).

6. Misner, C. W.: Astrophys. J. 151, 431 (1968).

7. Sachs, R. K.: Lectures at Brandeis Summer School (1968, to be published).

8. Lindquist, R. W.: Ann. Phys. (N.Y.) 37, 487 (1966).

9. Hawking, S. W.: Monthly Notices Roy. Astron. Soc. 142, 129 (1969).

10. Misner, C. W.: Phys. Rev. 186, 1319 (1969).

11. Partridge, R. B., Wilkinson, D.: Phys. Rev. Letters 18, 557 (1967).

12. Sandage, A.: Yearbook Carnegie Inst. Wash. 65, 163 (1966).

Dr. R. Matzner

Institute of Theoretical Astronomy

Madingley Road

Cambridge/England 